\title{
Spontaneous rupture of renal pelvis secondary to ureteral obstruction by urothelial tumor
}

\section{RUPTURA ESPONTÂNEA DE PELVE RENAL SECUNDÁRIA À OBSTRUÇÃO URETERAL POR TUMOR UROTELIAL} Daniel Alvarenga Fernandes ${ }^{1 *}$, Ana Laura Gatti Palma ${ }^{2}$, Ricardo Yoshio Zanetti Kido² ${ }^{2}$ Ricardo Hoelz de Oliveira Barros ${ }^{1}$, Daniel lahan Martins ${ }^{3}$, Thiago José Penachim ${ }^{3}$, Nelson Marcio Gomes Caserta ${ }^{4}$

${ }^{1}$ Radiologist, Assistant Physician, Department of Radiology, Faculty of Medical Sciences - State University of Campinas (FCM-Unicamp), Campinas, SP, Brazil ${ }^{2}$ Resident Physician, Department of Radiology, FCM-Unicamp, Campinas, SP, Brazil.

${ }^{3}$ Radiologist, Assistant Physician, Department of Radiology, FCM-Unicamp; Radiological Center of Campinas - Hospital Vera Cruz; Pontifical Catholic University of Campinas (PUCC), Campinas, SP, Brazil. ${ }^{4}$ PhD-Professor, Department of Radiology, FCM-Unicamp, Campinas, SP, Brazil.

Study conducted at the Department of Radiology, Faculty of Medical Sciences State University of Campinas (FCM-Unicamp), Campinas, SP, Brazil.

Article received: $10 / 6 / 2013$ Accepted for publication: $2 / 20 / 2014$

*Correspondence: Address: Department of Radiology of Faculty of Medical Sciences - State University of Campinas (FCM-Unicamp), Campinas, SP, Brazil. Rua Tessália Vieira de Camargo, 126, Cidade Universitária "Zeferino Vaz". Postal Code: $13083-887$ Campinas, SP - Brazil daniel_alvafer@yahoo.com.br daniel_alvafer@icloud.com

http://dx.doi.org/10.1590/1806-9282.60.05.006 Conflict of interest: none

\section{INTRODUCTION}

Partial spontaneous rupture of the upper urinary tract is rare and usually associated with nephrolithiasis. Other reported causes, apart from instrumentation and trauma, involve obstructive ureteral tumor in the pelvic cavity, retroperitoneal fibrosis, fluid overload, and pregnancy. We report a case of spontaneous rupture of renal pelvis secondary to ureteral obstruction caused by urothelial tumor, clinically suspected and evaluated by CT scans and MRIs, discussing the relevant findings for diagnosis.

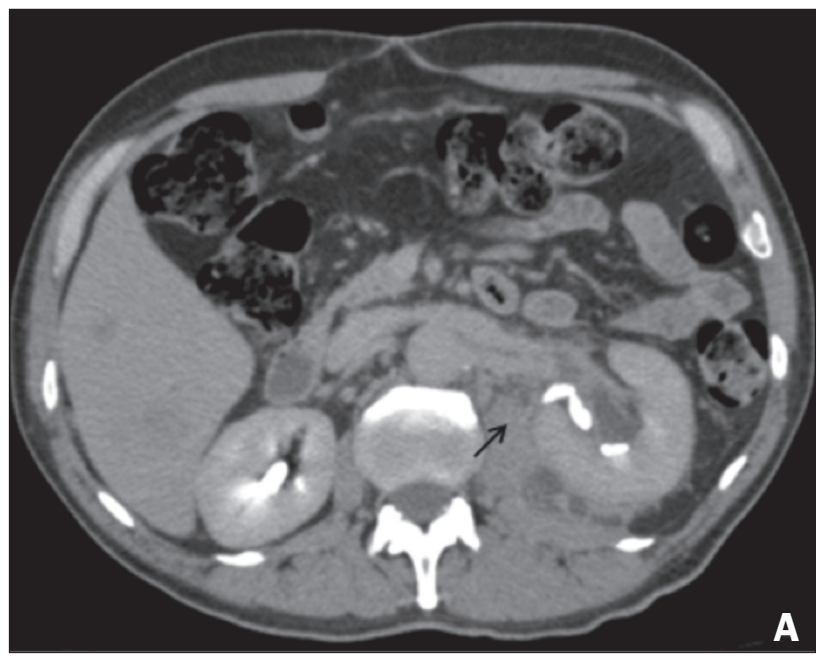

\section{Case report}

Male patient, 55 years, with a clinical picture of macroscopic hematuria and insidious lower back pain similar to cramps for about six months, which became stronger in recent months. The patient reports a fever, not measured. Denies trauma, previous surgery, history of urolithiasis, other complaints, and past illnesses. Former smoker (22 packs/year, having quit smoking for eight years). On physical examination, the patient showed good general health, being thin (body mass index of $17.2 \mathrm{Kg} / \mathrm{m}^{2}$ ), pallid $(1+/ 4$ ),

FIGURES 1A AND 1B Computed tomography, contrast-enhanced axial sections; excretory phase shows irregular parietal thickening of the left ureteropelvic junction, causing ureteral obstruction (black arrow) and moderate upstream pyelocaliceal dilatation (dashed arrow). Rupture of the relıal pelvis with contrast extravasation and formation of posterior perirenal urinoma is also observed (white arrow). 

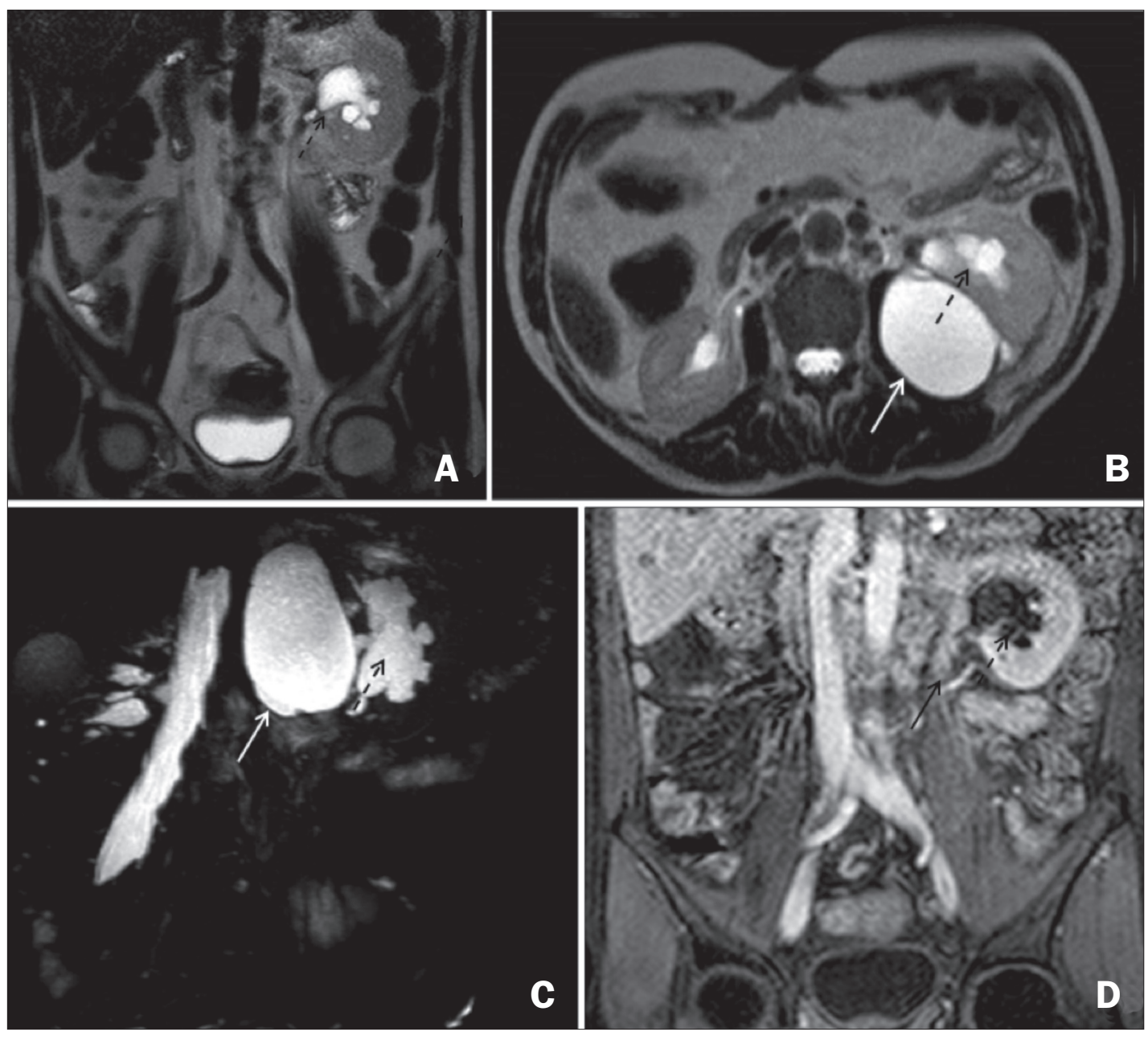

FIGURES 2A, 2B, 2C AND 2D MRI with T2-weighted images, coronal (2A) and axial (2B), three-dimensional MR urography (2C) and post-contrast T1 image (2D) revealing vascularized tumoral thickening of the proximal left ureter (black arrow), causing ureteral obstruction and moderate pyelocaliceal upstream dilation (dashed arrow). Associated rupture of the renal pelvis with contrast extravasation and formation of posterior perirenal urinoma also observed (white arrow), displacing the kidney anterolaterally.

hypohydrated (2+/4), acyanotic, anicteric, eupneic and afebrile. Abdomen with bowel sounds present and preserved, slightly painful on palpation in the left flank and iliac fossa. Painful lumbar fist percussion on the left.

Based on the clinical picture, we requested a contrasted multi-slice computed tomography (Figures $1 \mathrm{~A}$ and $1 \mathrm{~B}$ ) and subsequent complementation with MR urography - Uro-MRI (Figures 2A, 2B, 2C and 2D). Vascularized tumoral thickening of the proximal left ureter evident, causing ureteral obstruction and moderate pyelocaliceal upstream dilation. Associated rupture of the renal pelvis with contrast extravasation and formation of posterior perirenal urinoma is also observed, displacing the kidney anterolaterally.

Imaging diagnosis (with subsequent histopathological confirmation) led to the conclusion that it was a case of spon- taneous rupture of renal pelvis and formation of urinoma secondary to ureteral obstruction due to urothelial tumor.

\section{Discussion}

In the case described, the spontaneous rupture of renal pelvis was caused by urothelial tumor. The rupture diagnosis can be clinically suspected and confirmed by imaging. The clinical picture includes macro or microscopic hematuria (75\%), back pain (18\%), dysuria (6\%) and less frequently palpable mass, decreased appetite, weight loss, and may sometimes be asymptomatic. ${ }^{1-4}$

Urothelial tumors can affect any part of the urinary tract is relatively rare in the upper urinary tract (approximately $1 \%$ of all urothelial tumors), most frequently affecting patients in the sixth and seventh decades of life. The 
histological subtypes include transitional cell carcinoma (TCC) (90\%), squamous cell carcinoma (9\%), mucinous adenocarcinoma (less than 1\%), sarcomas, undifferentiated tumors and benign tumors (mesodermal inverted papilloma, and fibroepithelial polyp). Risk factors involve smoking, prolonged exposure to dyes such as aniline, petrochemical agents and tar, analgesic abuse (especially phenacetin), Balkan nephropathy, as well as factors that promote urinary stasis, as horseshoe kidneys. The use of cyclophosphamide, in turn, increases the risk of high-grade urothelial tumors. Chronic infection and calculi may also be associated with squamous cell carcinoma and mucinous adenocarcinoma. Schistosomiasis can be associated with the squamous cell carcinoma, and also seems to be associated with a greater degree of epigenetic changes in the epithelium of the genitourinary tract. ${ }^{4 \cdot 6}$

There is a clear need in patients with upper urinary tract tumors for imaging investigation of the entire urinary tract, due to possible synchronous bladder cancer in $2-4 \%$ of patients. Furthermore, the need for follow-up is well established, as $40-50 \%$ of patients with TCC will develop metachronous tumours involving the lower urinary tract. ${ }^{3-5}$

Urotomography (Uro-CT) performed with equipment with multiple rows of detectors (MDCT) is being increasingly used for evaluation of synchronous and metachronous tumors in the upper urinary tract and primary evaluation of hematuria. Imaging studies are also used to differentiate tumors and benign urothelial changes which might not require additional invasive assessment such as ureteroscopy and retrograde pyelography. ${ }^{7-9}$ The differential diagnosis of tumors in the upper urinary tract includes nephrolithiasis, blood clot, infection, tuberculosis, ectopic papilla, endometriosis and malakoplakia. Factors that help to reach a correct diagnosis include knowledge of the location, lesion density and pattern of impregnation, pericalicinal/periureteral opacification associated or not, and multiplicity of lesions. In the unenhanced phase of Uro-CT, TCCs are discreetly hyperdense compared to urine (15-30HU), unlike clots and stones (with respective densities at 40-80 HU and above 200HU). Since most of them are hypovascularized, they are discreetly impregnated by contrast (increase of 40-70HU), and to a lesser intensity than normal renal parenchyma, appearing in the excretory phase of the examination as fixed filling defects. ${ }^{5}$ In addition to technological improvements in MDCT, refinements in the tests' radiological orientation have probably contributed to increase the capacity to detect urothelial abnormalities. Different techniques have been used, such as abdominal compression, administration of intravenous saline solution, diuretics or both, "log-rolling" (asking patients to roll $360^{\circ}$ ) prior to excretory phase to maximize opacification and distention of the urinary tract.

On MRI, TCCs can appear hypointense or isointense compared to the renal parenchyma on T1- and T2-weighted sequences, making it necessary to use paramagnetic contrast agents in non-dilated urinary tract, being the TCCs less contrasted than the renal parenchyma. In patients with impaired renal function, static MR urography can help detect tumors of the upper urinary tract, especially in obstructed kidneys, since TCCs are usually hypointense compared to urine on T2-weighted images, which facilitates their detection when associated to hydronephrosis. The use of diffusion-weighted imaging (DWI) sequences associated with conventional T1- and T2-weighted sequences increases the accuracy and sensitivity of Uro-MRI. ${ }^{10,11}$

\section{Conclusion}

Although rare, spontaneous rupture of renal pelvis secondary to ureteral obstruction caused by urothelial tumor may be suspected clinically, and the detailed radiological analysis of tomographic images and MRI-guided sequences, including Uro-MRI, is of great importance for both diagnosis and follow-up of these patients.

\section{References}

1. Tripathi S, Ahmad A, Mathew VV. Spontaneous partial rupture of the upper renal tract. Case Report. Brunei Int Med J. 2011; 7 (4): 215-219.

2. Ashebu SD, Elshebiny YH, Dahniya MH. Spontaneous rupture of the renal pelvis. Australas Radiol. 2000; 44:125-7.

3. Kaplan M, Aktoz T, Atakan IH. A rare cause of acute flank pain: spontaneous rupture of the renal pelvis. Turkish Journal of Urology 2009; 35(3):256-259.

4. Inahara M, Kojima S, Takei K, Naito H, Kito H, Yamazaki K, et al. Two cases of spontaneous rupture of upper urinary tract caused by the primary ureteral or renal pelvic tumor: a case report. Acta Urologica Japonica. 2009; 55(1): 31-34.

5. Prando A. Tumores uroteliais. In: Muller CIS, D'Ippolito Giuseppe, Rocha AJ, series editors. Prando A, Baroni RH, associated editors. Urinário (Colégio Brasileiro de Radiology and Diagnostic Imaging; 5). Rio de Janeiro: Elsevier; 2013. p. 321-358

6. Gutiérrez MI, Siraj AK, Khaled H, Koon N, El-Rifai W, Bhatia K. CpG island methylation in Schistosoma- and non-Schistosoma-associated bladder cancer. Modern Pathology (2004) 17, 1268-1274.

7. Silverman SG, Leyendecker JR, Amis ES. What is the current role of CT urography and MR urography in the evaluation of the urinary tract? Radiology 2009; 250:309-323

8. Dillman JR, Caoili EM, Cohan RH, Ellis JH, Francis IR, Schipper MJ. Detection of upper tract urothelial neoplasms: sensitivity of axial, coronal reformatted, and curved-planar reformatted image-types utilizing 16-row multi-detector CT urography. Abdom Imaging 2008; 33:707-716.

9. Sadow CA, Wheeler SC, Kim J, Ohno-Machado L, Silverman SG. Positive Predictive Value of CT Urography in the Evaluation of Upper Tract Urothelial Cancer. AJR 2010; 195:337-343.

10. Yoshida S, Masuda H, Ishii C, Tanaka H, Fujii Y, Kawakami S, et al. Usefulness of Diffusion-Weighted MRI in Diagnosis of Upper Urinary Tract Cancer. AJR 2011; 196:110-116.

11. Padhani AR, Liu G, Koh DM, Chenevert TL, Thoeny HC, Takanara T, et al. Diffusion-weigheted magnetic resonance imaging as a cancer biomarker: consensus and recommendations. Neoplasia 2009; 11:102-125. 